\title{
Organisational Capabilities Required for Enabling Employee Mobility through Bring- Your-Own-Device Concept
}

\author{
B-Abee Toperesu \\ Information and Communication Technologies Services (ICTS), University of \\ Cape Town, South Africa \\ Jean-Paul Van Belle \\ Department of Information Systems, University of Cape Town, South Africa
}

\section{Abstract}

Background: Mobile device adoption is on the rise and people are increasingly using mobile devices as a part of their lives. Studies have shown that people can use mobile devices to perform their work duties from anywhere. Organisations are now exploring ways of enabling and supporting mobility for employees' mobile devices, including BYOD (Bring-Your-Own-Device) policies. Objective: The objective of this study is to identify the main capabilities required for enterprise mobility. Methods/Approach: This qualitative research study presents empirical results based on interviews with selected senior IS managers of large organizations. Results: The main findings of this study suggest that information security and mobile device management are among the main capabilities required for enterprise mobility. Conclusions: Enterprise mobility is an emerging field which has received very little research attention. More research in the field will help organisations make informed decisions on how to increase productivity, sales and efficiency while achieving employee satisfaction through enterprise mobility.

Keywords: mobility, productivity, use case, security, BYOD, Bring-Your-Own-Device, connectivity

JEL classification: $\mathrm{O} 33$

Paper type: Research article

Received: Nov 01, 2016

Accepted: Feb 26, 2017

Citation: Toperesu, B., Van Belle, J. P. (2017), "Organisational Capabilities Required for Enabling Employee Mobility through Bring- Your-Own-Device Concept", Business Systems Research, Vol. 8, No. 1, pp. 17-29.

DOI: 10.1515/bsrj-2017-0002

\section{Introduction}

The advances in mobile technologies have enabled exciting new possibilities for individuals and organisations. The considerable developments in mobile hardware, software and networks contribute greatly to mobile collaboration and communication. Mobile hardware available on the market includes tablet 
computers and smartphones, while advanced applications have been developed for these devices based on the iOS and Android platform. Advanced mobile networks such as $3 G$ and LTE allow seamless connection of these devices with fast data transfer rates enabling advanced communication software such as Skype video calling to work seamlessly. These developments in mobile technologies have made mobile devices sophisticated and powerful to the extent that their performance nearly equals that of desktop computers (Ghosh et al., 2013).

As a consequence, the traditional workplace is changing as individuals now perform their work duties using mobile devices at home, from remote sites or while on the go. Mobile technologies enable workers to work and collaborate while on the move (Kietzmann et al., 2013). However, enterprise mobility has not received much research to date (Sørensen, 2014).

The purpose of this research study is to come up with an empirically validated list of 'must-have' capabilities for enterprise mobility. Qualitative data was collected and analysed and final conclusions drawn from it.

This study seeks to contribute new insights on enterprise mobility to both theory and practice. Organisations seeking to optimise enterprise mobility can use the research findings as a reference. Researchers may find the proposed model of factors, capabilities and impacts useful for research into enterprise mobility and related fields.

The paper first presents a literature review outlining the benefits, issues and impacts of enterprise mobility; and introduces the theoretical framework. The paper then discusses the methodology, followed by a detailed analysis of the empirical data. It concludes with recommendations, limitations and suggestions for future research.

\section{Background}

The key terms used in this research are Capability and Enterprise Mobility. Several definitions of capability exist depending on the context in which it is being used (Harris, 2007). This research followed the definition that "organisational capability refers to an organisational ability to perform a co-ordinated task, utilizing organisational resources, for the purpose of achieving a particular end result" (Helfat, 2003, p. 1). Enterprise mobility is when work interaction is mobilized using mobile and ubiquitous information technology (Sørensen, 2014). Enterprise mobility can therefore be defined as "the use of mobile IT for the accomplishment, coordination and management of organizational activities" (Sørensen, 2011, p. 476).

\section{Enterprise Mobility Management}

Enterprise Mobility Management is designed to address the risk of unauthorised access of organisational information on mobile devices by implementing some level of control on such devices without compromising the privacy of the device owner (Peraković et al., 2014). In achieving this goal, there are methods developed and used to separate and secure business data and private data on a mobile device. These methods fall under the term Mobile Content Management. The methods include Mobile Device Management (MDM), Mobile Application Management (MAM), Mobile Information Management (MIM) and Mobile Email Management (MEM). These methods share the common purpose of managing and securing a mobile device's data and applications. 
Using wireless communication technologies such as WiFi and over-the-air (OTA), Mobile Device Management, "supports centralized control of an entire fleet of smartphones and other mobile devices by applying and ensuring pre-defined configuration settings using an app on the smartphone" (Beimborn et al., 2013, p. 2). The app that is used to apply configurations on the MDA system is called an MDM agent. Security settings such as password length and certain device functionality are governed by the MDA agent (Hemker, 2012).

Adopting MDM systems helps enhance the security of mobile devices; however, if the system is exploited, mobile devices and the data on them may be compromised. A study by Rhee et al. (2013) identified and listed all possible threats against an MDA system through analysis of threat agents, assets and adverse actions. Developers need to consider all these possible threats in order to secure and prevent the mobile devices from being exploited.

Organisational content is mainly delivered through mobile applications to users. Mobile Application Management is, described by Winthrop (as cited in Beimborn et al., 2013, p. 2) who states that "MAM focuses on managing the life cycle of an app from development, procurement, distribution, configuration, and update to the removal of an app." Some of the essential functions of MAM are application containerisation, support for application-specific containers, secure access to email and disabling copying of its contents and copying of files between applications (Peraković et al., 2014).

Proceeding from MAM is Enterprise App Store (EAS). EAS is a platform for internal organisational distribution of mobile apps limited to business apps. The firm predefines or pre-qualifies apps in the organisation's context which employees can use and put them in a container (Beimborn et al., 2013).

With Mobile Information Management, the focus is shifted to securing critical corporate information. The main purpose of MIM is to store enterprise information on a central location, possibly a private cloud, then share it with different endpoints in a secure manner (Eslahi et al., 2014). These endpoints could be a limited number of devices which can access or manage the encrypted data. It should be noted, however, that MIM on its own is not a defence against hacking or malware attacks but is a way of managing access to sensitive corporate data by mobile devices (Allan, 2014).

\section{Enterprise Mobility Benefits}

There are benefits to the organisation; these include cost savings, competitive advantage, a high return on investment, etc. Benefits to the employee include flexibility, increased productivity, etc., while customer or client benefits include convenience and efficiency. Basole (2007a) argues that there are five types of enterprise mobility benefits: strategic benefits - which include creating a competitive advantage through aligning business goals with strategies; informational benefits i.e. easier access to correct information; transactional benefits - cutting operational and staff costs; enterprise transformation - enhanced structure and procedures; and business value benefits - which in cooperates all these aforementioned benefits.

\section{Enterprise Mobility Impacts}

Enterprise Mobility (EM) can have a number of impacts on business; positive impacts may also be termed benefits. A sharp increase in employee productivity may perhaps be the greatest impact an organisation may experience, with $75 \%$ of enterprises adopting EM reporting increased employee productivity (Kumar, 2012). 
Tech savvy employees working from mobile devices are more likely to continue working outside of work hours and thus have quicker turnaround times on their assigned tasks (Pillay et al., 2013).

Direct access to inventory levels and pricing, ability to access and update customer profiles, and carrying out transactions in real-time enables elimination of redundant procedures which in turn shortens sales cycles and increases sales (Ortbach et al., 2014). Using ERP mobile applications optimises real time access to customer orders and inventory levels for sales staff while management has access to time critical performance indicators through dashboards.

Cost savings, and thus an increased Return on Investment (ROI), can be realised by an organisation when a shift from purchasing expensive desktop computing equipment is replaced by cheaper mobile devices (Basole, 2007b). Significant reductions in IT support costs can also be realised when employees use their own devices. Employees would then manage their own devices or get support from their vendor, which in turn reduces IT administration by the organisation.

EMM also impacts on various intangible aspects such as employee and customer satisfaction, although measuring such impacts has proven to be difficult due to a lack of assessment tools (Vuolle, 2011). However, it is acknowledged that some positive factors in EM do contribute to the overall satisfaction of the user or customer. Carden (2007) argues that the ability to check the status of a request using a mobile application like an ERP app increases customer assurance and overall satisfaction. When a customer of an insurance company is able to file a claim using a mobile app, the result is process improvement and increased efficiency as well as customer satisfaction (Giessmann et al., 2012).

\section{Enterprise Mobility Challenges}

As smartphones and tablets become common in the workplace, securing corporate information on these devices has become a great concern to enterprises. The main challenge is to put in place appropriate security policies while exploiting the usability of consumer applications (Sammer et al., 2013).

Another challenge is lack of control of the device. When the employee owns the device, it is up to them to keep the device physically secure from use or access by others and, in the event that the device is lost or stolen, the device will become a security threat to the organisation. IT practices and policies, password protection and remote wipe need to be enforced; they can also be intentionally averted by employees for various reasons (Pillay et al., 2013).

Developing compatible applications has become a headache for developers trying to produce applications that work on as many mobile devices as possible. Fragmentation of mobile device platforms presents challenges such as app support, enforcing security updates, monitoring and provisioning. In an attempt to address this issue, some vendors are offering cross platform enterprise app stores as a solution since they only need a web browser (Balakrishnan et al., 2015).

\section{Enterprise Mobility Enablers}

According to Basole (2008), mobile applications are one of the most significant enablers of enterprise mobility. He notes that mobile work specific applications have been developed in many cases by encompassing the existing business applications into the mobile sphere. This has led to the development of mobile app stores. App stores can be described as "digital platforms that provide users a central location to 
effectively browse, purchase, download, and update their mobile applications" (Jin et al., 2014, p. 1).

Advancements in mobile network technologies have also enabled not only voice transfers but also data transfers. Widespread availability of $3 G$ and $4 G$ mobile spectra and advanced technologies like LTE, bandwidth and browsing capabilities are drastically improving on mobile devices (Kumar, 2012).

The introduction of large screen smartphones called 'phablets', brought about more possibilities of users using these devices to access cooperate information. Davis (2014) notes that users interact with these devices in different and unconsidered ways whether they are controlled or managed. He further notes that the number of employees using mobile devices to work is increasing--a confirmation of how mobile device advancements is enabling enterprise mobility.

BYOD (Bring Your Own Device)is when employees use non-corporate owned devices including their own software to access company resources within or outside the environment of their organisation (Ghosh et al., 2013). BYOD is one of the major enablers of enterprise mobility, as organisations do not have to purchase and provide any mobile devices for its employees. The number of organisations taking advantage of BYOD is increasing globally with countries such as Spain, Malaysia and Brazil reporting up to $80 \%$ adoption (Eslahi et al., 2014).

\section{Conceptual Model for Organisational Capabilities}

The TOE (Technology-Organisation-Environment) framework and other technology frameworks such as the Diffusion of Innovation (DOI) available in literature tend to mainly focus on the adoption of IT (Kabanda et al., 2015, Oliveira et al., 2011, Molla et al., 2005). No framework is available for measuring IT capabilities at firm level. However, in cases where available frameworks fail to address the research requirements, there is evidence of available studies which combined theories to address this gap (Oliveira et al., 2011). Based on the TOE framework, this research used the conceptual framework below to identify the essential capabilities for improving enterprise mobility.

Figure 1

Conceptual model for research based on the TOE framework

Factors/Enablers (How) Capabilities (What)

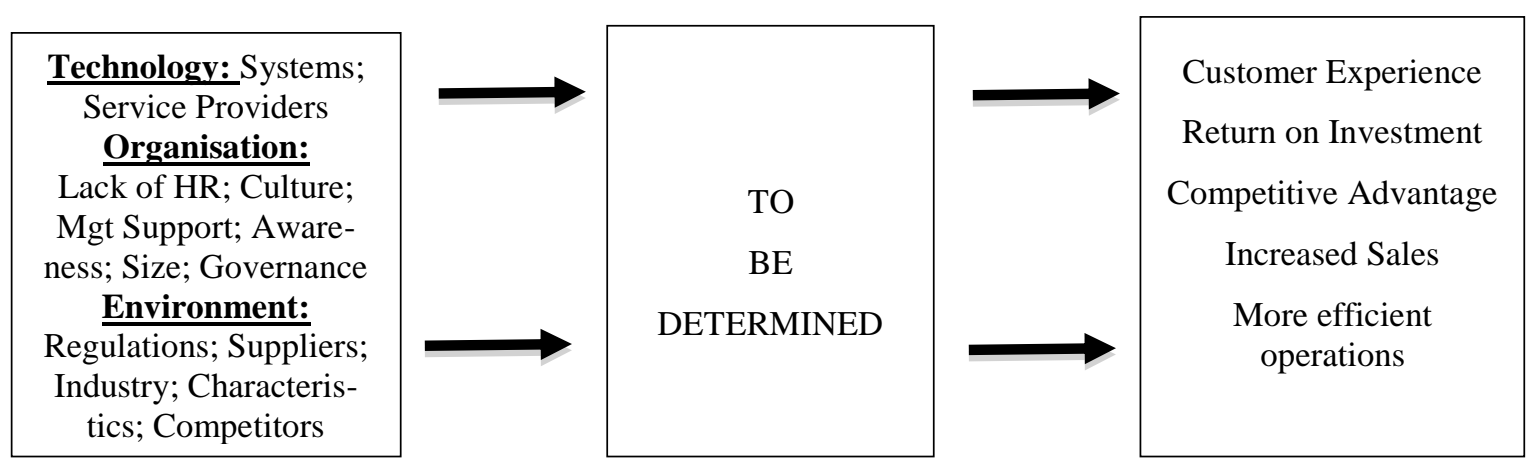

Source: Tornatzky et al., 1990

This study used the Technology-Organisation-Environment (TOE) framework (Tornatzky et al., 1990) to identify the capabilities for improving enterprise mobility (Figure 1). The model has three components. The first component is the factors that 
influence certain capabilities. The factors are grouped under three contexts i.e. technological factors, organisational factors and environmental factors. These factors would have a positive or negative effect on the capabilities listed in the middle section. The second component is the capabilities which will be determined during data collection. These capabilities will in turn influence certain outcomes, also to be determined in this research.

\section{Methodology}

The main research question was "What are the must-have capabilities for enterprise mobility?" The secondary questions were:

1. What are the technologies, organisational and environmental factors that influence enterprise mobility?

2. What impacts or outcomes does enterprise mobility have?

This research study is qualitative and used a post-positivist philosophy. Unlike positivism which works with an observable social reality and provides law-like generalizations as its findings (Saunders et al., 2009), the post-positivism approach recognises "that all cause and effect is a probability that may or may not occur" (Creswell, 2013, p 23). This research was cross sectional. Our population was large organisations, which befit the term enterprise. Our findings are not necessarily generalizable to small and medium-sized enterprises.

Five organisations were selected from each of the following sectors: Insurance, Accounting, Auditing, and Whole Sale and Retail Industry. The reason of this selection is for diversification. Purposeful sampling was used in selecting these organisations in order to find senior managers who understood the study phenomenon best. Data was collected using semi-structured interviews. Questions were prepared beforehand and served as a guideline so as to cover the entire required topic field. New questions emerged during the conversation, giving the interviewee an opportunity to further elaborate their responses. Open-ended questions were used to allow the interviewee to fully express himself or herself, thereby providing rich data.

Interviews were recorded and transcribed; thereafter, thematic analysis and coding was used to uncover themes and patterns in the data. Thematic analysis makes use of coding themes from the data using NVivo. NVivo is a qualitative data analysis tool, which facilitates shaping, and making sense of unstructured data by producing trends and enabling hypothesis construction and demonstration.

Candidates participated in this research on a voluntary basis. The details of participants and their organisations were treated as highly confidential and aliases are used where necessary.

\section{Results}

Interviews were conducted with 5 senior managers and 1 enterprise mobility consultant (referred to as P1 to P6) as shown in Table 2.

The data was transcribed and analysed using thematic analysis. The following section presents the themes that came up from the interviews, grouped into the categories of Factors, Capabilities and Impacts. 
Table 2

Interview Participants

\begin{tabular}{cll}
\hline Participant & Industry & Job Title \\
\hline P1 & Accounting & Senior Manager IT \\
P2 & Retail & Senior IS Manager \\
P3 & Accounting & Service Delivery manager \\
P4 & Insurance & Mobility Architect \\
P5 & Wholesale & IT Manager \\
P6 & IT Service & Mobility Consultant \\
\hline
\end{tabular}

Source: Authors' calculation

\section{Adoption Factors}

From our theoretical framework, we have technological, organisational and environmental factors. The following are the themes that came up under each section.

The existing themes that were originally listed in the theoretical framework were technical systems and service providers. However, during the analysis, none of these themes came up. Instead the theme that came up under technology was hardware as mentioned by one participant: "So you need hardware with good battery life, good connectivity options, WiFi, 3G, and as I have mentioned the right form factor screen size to be able to work" (P1). This assertion suggested that there is a need to have the right tools in place in terms of the required hardware that will run, maintain, support and manage the mobile devices.

The findings suggest that organisational culture seemed to be a big issue across all the participants' organisations, as stated by the participants: "I think the most important factor is the internal cooperate culture" (P1); "People are very used to having offices, having computers and sitting doing work. Now we are telling them listen forget about that" (P2); "Some people are very paper driven so they don't... almost have resistance to change..." (P5); However, other participants did not agree as they state that users already have a culture of technology; And "A lot of the users (at...) already have a culture of technology" (P4). Organisational culture then seems to be a problem to the older generation of staff members who may struggle to embrace new technologies.

The only environmental theme that emerged was government regulation, although this does not seem to affect enterprise mobility much as mentioned by the participants: "Basically the factors we will have to consider is that also the POPI act is one factor (that is the Protection of personal information) so we can't, we can not just have e-mail on the phone with normal technology because there is no security measures put in place. So we have to consider the structured and unstructured data how it's handled how it's shared how it's stored where it's stored and those kind of things yah" (P4). However, Participant 1 did not agree as he states that, "Those can be complied with and still have mobility as a big aspect of our business so there is no regulations as far as I know or any other factors really that affects us" (P1).

\section{Capabilities}

This section presents the major capabilities that emerged during the interview process, based on conversation density.

At the core of enterprise mobility is connectivity. Without connectivity, the whole concept of enterprise mobility is disabled as emphasised by this participant: "I think 
one of the biggest challenges that we have experienced is the connectivity so when people are trying to work offsite or remotely and there is a slow or unstable connection back to the office that causes the biggest issues so then people aren't able to work...So that's the single biggest issue or barrier to enterprise mobility for us (P1).

The ability to have the right hardware for enterprise mobility came out as one of the dominant themes. This includes the mobile devices themselves and their ability to connect to a network as mentioned by the participants. "Second most important is the hardware itself. So you need hardware with good battery life, good connectivity options, WiFi, 3G, and as I have mentioned the right form factor screen size to be able to work" (P1). "Laptops that we issue [have] built in $3 \mathrm{G}$ cards and we supply the users with sim cards and make sure you are always connected to the internet (P1).

Most participants agreed that in order to have buy-in for enterprise mobility from users, you need to have a change management strategy. Most organisations are dealing with people who are used to their old way of thinking and working, and when new ways of doing the same tasks are introduced, proper change management is essential to achieve the main goals and purposes of enterprise mobility as mentioned by these participants. "So I think the change management is critical. Making sure that people understand why you are doing it and it is there to make their lives easier" (P2).

The need for a proper device management setup was agreed upon by most participants interviewed. This is in line with the literature, which also placed emphasis on the need for a mobile device management setup to ensure secure enterprise mobility. The main purpose of mobile device management, as discussed by the participants, is to have a level of control on the devices' functions and usage with the purpose of ensuring the organisation's systems are secured from outside threats, internal abuse and malicious activities.

The need to have secure enterprise mobility was raised by most participants. This theme also relates to the above previous discussion on Mobile Device Management as MDM works to ensure security. "If you look at South Africa and you look at Kenya, to them enterprise mobility is not something that they discussed because they believe everyone to be mobile and people in organisations has mobile but are they mobile secure? Can they do remote wipe?" (P6). "Enterprise mobility it's about basically securing information on mobile devices" (P4).

Most participants feel there is a need to have mobile applications made specifically to run on mobile devices. This may include optimising the current applications such as e-mail to run on mobile.

Most participants placed emphasis on choosing the right use cases as being very important. "You need to understand where they need mobility and what they get. You need to have a requirement, and that requirement needs to be understood" (P6). "But I think more importantly on all those is picking the right use cases I think is what you call them. Start there first because the danger is you start with the technology and then you look at the processes" (P2). Letting technology drive mobility has a negative impact of having the systems and applications which do not really serve any purpose. This would in turn affects the usability of the technology.

\section{Impacts}

This section presents a list of the impacts, outcomes and benefits that are as a result of an organisation's enterprise mobility.

Most participants were of the view that, to them, enterprise mobility has brought a significant increase in productivity. "I think people have become more productive 
because of mobility" (P3). "What enterprise mobility on the mobile devices has enabled us to do is to increase productivity" (P1). "I think the biggest impact is gotta be on store productivity" (P2). This is in line with findings from the literature, which listed increased productivity as a major benefit. Increased productivity is due to the flexibility that comes with working from anywhere.

Increased efficiency or effectiveness also came up in the interviews. Participants mentioned that with mobility comes efficiency in doing and carrying out tasks, as emphasized by participant 5: "I mean that was the main thing to be a little more efficient" (P5). This is in line with the literature findings that enterprise mobility allows work to be done more efficiently, resulting in significant time savings.

Another benefit is increased sales. "So there is a full impact across the system. It starts with store efficiency and productivity that actually leads to increase in sales" (P2). It is worth noting that the increase in sales is as a result of the other impacts like efficiency and productivity as mentioned by the participant.

Satisfaction is dependent on successful change management. Once the idea has been properly sold to the staff on how it's all going to work and how it will make their jobs and lives easier and they actually realise the benefits of using such, their satisfaction increases. "I think you have to tell your staff why you are doing what you are doing and explain to them how the process is going to change and why it's going to change and how it's going to make their jobs easier" (P6).

\section{Discussion}

The data analysed from the interviews provided the researchers with the following findings. Most of the factors that were provided in the theoretical framework were not validated by the data. However, several new factors were uncovered (Figure 2).

Under technological factors, technology systems and service providers were not validated by the data. However, hardware came up as a new theme and is an important factor, as emphasised by participants. The hardware referred to includes the file servers and the mobile devices themselves that lie at the root of mobility.

Figure 2

Organisational Mobility Capability Model

\begin{tabular}{|c|c|c|}
\hline Factors/Enablers (How) & Capabilities (What) & Impacts/Outcomes (Why) \\
\hline $\begin{array}{c}\text { Technology } \\
\text { Hardware } \\
\text { Organisation } \\
\text { Culture }\end{array}$ & $\begin{array}{c}\text { Tools } \\
\text { Change } \\
\text { Management } \\
\text { Connectivity }\end{array}$ & $\begin{array}{l}\text { Increased Sales } \\
\text { Increased } \\
\text { Productivity }\end{array}$ \\
\hline
\end{tabular}

Source: Authors' work

The organisational factors that most affect enterprise mobility are culture. The other factors were not validated. However, it can be noted that lack of awareness is closely associated with culture as raised by Participant 4: "It's a matter of how to do those things and there is always requests from users to see how they can counter from mobile devices" (P4). 
The findings show that under environmental factors, only two factors were considered to affect enterprise mobility: government regulations, such as the POPI act, and a new theme network infrastructure. Interestingly, the other factors originally identified were not mentioned, such as suppliers and competitors.

Capabilities were identified from the interview data. Some capabilities themes came out dominant and were in line with the literature. Although Use Cases and Change Management did not receive many references in the interviews, the emphasis that was placed on them is of much significance.

Taking a look at impacts, the findings reveal four main impacts as a result of enterprise mobility. These are increases in satisfaction, sales, productivity and efficiency. User satisfaction and increased productivity were new themes that came up. The major impact highlighted by participants was increased productivity. This is due to the ability to work from 'anywhere'. Other impacts such as customer experience, return on investment and competitive advantage were not validated.

The list of capabilities that has been presented in the middle block of the framework all came from the data, which was analysed. New themes of capabilities were identified and analysed as they came up from the interviewed participants. An inductive process was then applied to complete the framework's constructs and initially the block was blank as there were no capabilities identified in literature by which we could validate. It is important to note that some capabilities themes came out dominant and were in line with the literature. The dominant themes that were identified based on conversation density in terms of the number of times the theme was mentioned in the interview are shown in the Table 3.

Table 3

Summary of Themes

\begin{tabular}{lllllll}
\hline & P 1 & P 2 & P 3 & P 4 & P 5 & P 6 \\
\hline Security & & 1 & 1 & 2 & 1 & 2 \\
MDM & 2 & 2 & 1 & 2 & 1 & \\
Connectivity & 5 & 1 & 7 & 1 & 1 & 2 \\
Use Cases & & 1 & & 1 & & 2 \\
Change Management & & 1 & & & & 3 \\
\hline
\end{tabular}

Source: Authors' calculation

Although Use Cases and Change Management did not receive much reference in the interviews, the emphasis that was placed on them is of much significance.

"You need to understand where they need mobility and what they get...You need to have a requirement, and that requirement needs to be understood"(P6). "I think the change management is critical. Making sure that people understand why you are doing it and it is there to make their lives easier" (P2).

Based on conversation density and emphasis, the capabilities which came up top are Security, Mobile Device Management, Right Use Cases, Change Management and Connectivity.

Taking a look at impacts, the findings reveal that there are four main impacts that are as a result of enterprise mobility. These are increased satisfaction, sales, productivity and efficiency. User satisfaction and increased productivity were new themes that came up. The major impact highlighted by participants being increased productivity. This is due to the ability of to work from 'anywhere'. Other impacts such as customer experience, return on investment and competitive advantage were not validated 


\section{Conclusion}

The purpose of this research was to determine the main capabilities for enterprise mobility. We also investigated what technological, organisational and environmental factors influence enterprise mobility and what impact or outcome enterprise mobility has.

The most important capabilities required for enterprise mobility which were uncovered in our empirical study were:

- Security- the ability to secure information being transmitted to and from mobile devices.

- Mobile Device Management: the ability to manage the mobile devices issued to staff. This includes the remotely wiping of the devices.

- Right Use Cases. An organization should be able to choose the Right Use Cases on which services and processes to offer on mobile devices.

- Change Management: the ability to effectively manage the change in shift to enterprise mobility.

- Connectivity: the ability to offer or operate in an environment where the mobile devices could be connected to a network.

In answering the secondary questions, the following findings were discovered. The factors that affect enterprise mobility are: hardware availability, culture and/or awareness, government regulations and network infrastructure. The most important impacts or outcomes of enterprise mobility to an organisation are user satisfaction, increased sales, productivity and efficiency.

The research findings are of significant importance to organisations seeking to either adopt an EM strategy or to organisations currently employing an EM strategy but needing to improve it. This list of must-have capabilities acts as a reference point to organisations as to what is it they need for EM.

The major limitation for the research was the small sample size. A larger sample size might have provided much richer data and possibly revealed other factors, capabilities and impacts. Also, since most of the organisations were mainly based in South Africa, the findings might not be generalizable to organisations worldwide.

Future research may wish to look at a particular industry in an effort to have a more accurate set of capabilities for enterprise mobility for that particular industry type. Also, an extension of this research in other country contexts is necessary in order to determine the generalizability of our findings.

\section{References}

1. Allan (2014), "Mobile Information Management", available at: http://www.digitalfuturetoday.com/2014/02/25/mobile-informationmanagement/ (27 April 2015)

2. Balakrishnan, N., Aziz, M., Ibrahim, F. (2015), "Enterprise App Store for Cross Platforms", International Journal of Future Computer and Communication, Vol. 4 No. 2, pp. 147-151.

3. Basole, R. C. (2008), "Enterprise mobility: Researching a new paradigm", Information Knowledge Systems Management, Vol. 7 No. 1-2, pp. 1-7.

4. Basole, R. C. (2007a), "Strategic planning for enterprise mobility: A readinesscentric approach", in 13th Americas Conference on Information Systems, AMCIS 2007, Association for Information Systems, Keystone, USA, pp. 3967-3979.

5. Basole, R. C. (2007b), "The Emergence of the Mobile Enterprise: A Value-Driven Perspective", in International Conference on the Management of Mobile Business (ICMB 2007), IEEE. 
6. Beimborn, D., Palitza, M. (2013), "Enterprise App Stores for Mobile Applications Development of a Benefits Framework", available at:

http://aisel.aisnet.org/amcis2013/EndUserlS/GeneralPresentations/15 (13 April 2015)

7. Carden, P. (2007), "Enterprise mobility", Enriching Communications, Vol. 1 No. 2, pp. 1-6.

8. Creswell, J. W. (2013). "Qualitative Inquiry \& Research Design", SAGE.

9. Davis, L. (2014), "Mobile Practices and the Increasing Individuation of Workplace", available at:

http://aisel.aisnet.org/cgi/viewcontent.cgi?article=1214\&context=ecis2014 (20 April 2017)

10. Eslahi, M., Naseri, M. V., Hashim, H., Tahir, N. M., Saas, E. H. M. (2014), "BYOD: Current state and security challenges", in 2014 IEEE Symposium on Computer Applications and Industrial Electronics (ISCAIE), IEEE, pp. 189-192.

11. Ghosh, A., Gajar, P. K., Rai, S. (2013), "Bring Your Own Device (BYOD): Security Risks and Mitigating Strategies", Journal of Global Research in Computer Science, Vol. 4 No. 4, pp. 62-70.

12. Giessmann, A., Stanoevska-Slabeva, K., de Visser, B. (2012), „Mobile Enterprise Applications--Current State and Future Directions", in 2012 45th Hawaii International Conference on System Sciences, IEEE, pp. 1363-1372.

13. Harris, R. (2007), "Unravelling the notion of organisational capability: What do writers say it is and VET providers think it is?", University of South Australia.

14. Helfat, C. E. (2003), "Stylized facts regarding the evolution of organizational resources and capabilities", in Helfat, C. E. (Ed.), The SMS Blackwell handbook of organizational capabilities: Emergence, development, and change, WileyBlackwell.

15. Hemker, T. (2012), "„Ich brauche das!" - Mobile Geräte im Unternehmenseinsatz", Datenschutz und Datensicherheit - DuD, Vol. 36 No. 3, pp. 165-168.

16. Jin, L., Deng, X., Lea, B. (2014), "An Explorative Study of Mobility Adoption in the Enterprise", available at:

http://aisel.aisnet.org/cgi/viewcontent.cgi?article=1038\&context=amcis2014 (8 April 2015)

17. Kabanda, S. K., Brown, I. (2015), "E-Commerce Enablers And Barriers In Tanzanian Small And Medium Enterprises", The Electronic Journal of Information Systems in Developing Countries, Vol. 67 No. 7, pp. 1-24.

18. Kietzmann, J., Plangger, K., Eaton, B., Heilgenberg, K., Pitt, L., Berthon, P. (2013), "Mobility at work: A typology of mobile communities of practice and contextual ambidexterity", The Journal of Strategic Information Systems, Vol. 22 No. 4, pp. 282-297.

19. Kumar, A. (2012), "Enterprise Mobility Strategy-Should Enterprises Care?", Infosys Labs Breifing, Vol. 10 No. 1, pp. 35-48.

20. Molla, A., Licker, P. S. (2005), "eCommerce adoption in developing countries: a model and instrument", Information \& Management, Vol. 42 No. 6, pp. 877-899.

21. Oliveira, T., Martins, M. (2011), "Literature Review of Information Technology Adoption Models at Firm Level", The Electronic Journal Information Systems Evaluation, Vol. 14 No. 1, pp. 110-121.

22. Ortbach, K., Brockmann, T., Stieglitz, S. (2014), "Drivers for The Adoption of Mobile Device Managemnet in Organisations", available at: http://aisel.aisnet.org/ecis2014/proceedings/track16/10 (13 April 2015) 
23. Peraković, D., Husnjak, S., Cvitić, I. (2014), "Comparative analysis of enterprise mobility management systems in BYOD environment", available at: http://www.fpz.unizg.hr/ikp/upload/perakovic_husnjak_cvitic.pdf (8 April 2015)

24. Pillay, A., Diaki, H., Nham, E., Senanayake, S., Tan, G., Deshpande, S. (2013), "Does BYOD increase risks or drive benefits?", available at: http://minervaaccess.unimelb.edu.au/handle/11343/33345 (8 April 2015)

25. Rhee, K., Won, D., Jang, S. W., Chae, S., Park, S. (2013), "Threat modeling of a mobile device management system for secure smart work", Electronic Commerce Research, Vol. 13 No. 3, pp. 243-256.

26. Sammer, T., Brechbühl, H., Back, A. (2013), "The New Enterprise Mobility: Seizing the Opportunities and Challenges in Corporate Mobile IT", in AMCIS 2013 Proceedings, pp. 1-8.

27. Saunders, M., Lewis, P., Thornhill, A. (2009). "Research methods for business students", Financial Times, Prentice Hall.

28. Sørensen, C. (2014), "Enterprise Mobility", available at: http://digitalinfrastructures.org/publications/Sorensen2012-CSChapterPreprint.pdf (24 March 2015)

29. Sørensen, C. (2011), "Enterprise Mobility: Tiny Technology with Global Impact on Work", Palgrave Macmillan.

30. Tornatzky, L. G., Fleischer, M. (1990), The process of technology innovation, Lexington, Lexington Books.

31. Vuolle, M. (2011). "Measuring Performance Impacts of Mobile Business Services from the Customer Perspective", available at:

https://tutcris.tut.fi/portal/files/5471742/vuolle.pdf (20 April 2017)

\section{About the authors}

B-Abee Toperesu is an Information Technology consultant and is doing his Master of Commerce degree in Information Systems at the University of Cape Town. With over 10 years' experience in Information and Communication Technology ranging from training and development, web development and IT Support, B-Abee is currently offering consultancy services at the University of Cape Town's IT service desk which offers technical support to staff and students. B-Abee's research interests are in mobile device adoption and use for development (M4D). He can be contacted at batops@gmail.com

Jean-Paul Van Belle is a professor in the Department of Information Systems at the University of Cape Town and Director of the Centre for IT and National Development. His research areas are the adoption and use of emerging technologies in developing world contexts including mobile, cloud computing. His passions are ICT4D and adoption of ICTs by small organisations. He has over 170 peer-reviewed publications including 25 chapters in books and about 40 refereed journal articles. He has been invited to give a number of keynote presentations at international conferences and holds an honorary professorship at Amity University. He currently supervises almost 20 Masters and PhD students and has graduated many more. Jean-Paul can be contacted at Jean-Paul.VanBelle@uct.ac.za 